\title{
On the Early Diagnosis of Alzheimer's Disease from EEG Signals: A Mini-Review
}

\author{
Justin Dauwels $^{+\dagger}$, François-Benoît Vialatte*, and Andrzej Cichocki* \\ ${ }^{+}$Laboratory for Information and Decision Systems (LIDS), Massachusetts Institute \\ of Technology (MIT), Cambridge, MA \\ ${ }^{\dagger}$ Amari Research Unit, RIKEN Brain Science Institute, Saitama, Japan \\ * Laboratory for Advanced Brain Signal Processing, RIKEN Brain Science Institute, \\ Saitama, Japan \\ justin@dauwels.com \\ \{fvialatte, cia\}@brain.riken.jp
}

\begin{abstract}
In recent years, various computational approaches have been proposed to diagnose Alzheimer's disease (AD) from EEG recordings. In this paper, we review some of those approaches, and discuss their limitations and potential.
\end{abstract}

\section{Introduction}

Alzheimer's disease (AD) is a common neurodegenerative disorder that affects more than $10 \%$ of Americans over age 65 , and nearly $50 \%$ of people older than 85 ; it is estimated that the prevalence of the disease will triple within the next 50 years [1]. No cure for Alzheimer's disease has been developed yet, but a number of medications are believed to delay the symptoms (and perhaps causes) of the disease. The progression of the disease can be categorized in four different stages. The first stage is known as Mild Cognitive Impairment (MCI), and corresponds to a variety of symptoms (most commonly amnesia) which do not significantly alter daily life. Between 6 and $25 \%$ of people affected with MCI progress to AD every year. The next stages of Alzheimer's disease (Mild and Moderate AD) are characterized by increasing cognitive deficits, and decreasing independence, culminating in the patient's complete dependence on caregivers and a complete deterioration of personality (Severe AD) [2].

Early diagnosis of Alzheimer's disease, and in particular diagnosis of MCI and Mild AD, is important, since medications are most effective at an early stage of the disease. Diagnosis of Alzheimer's disease is hard, however, and symptoms are often dismissed as normal consequences of aging. Diagnosis is usually performed through a combination of extensive testing and eliminations of other possible causes. Psychological tests such as Mini Mental State Examinations (MMSE), blood tests, spinal fluid, neurological examination, and increasingly, imaging techniques are used to help diagnose the disease [3].

In the last years, several research groups have started investigating the potential of electroencephalograms (EEGs) for diagnosing AD. Since EEG recording systems are inexpensive and (potentially) mobile, EEG may potentially be used as a tool to screen a large population for the risk of $\mathrm{AD}$.

In this paper, we review several state-of-the-art signal processing methods to detect perturbations in EEG signals caused by AD (Section 2). We assess 
the potential and limitation of such computational approaches (Section 3). At the end of the paper, we briefly address future challenges and open problems (Section 4). This paper is probably one of the few studies in recent years to provide an overview and critical assessment of various state-of-the-art signal processing methods for diagnosing AD from EEG signals.

\section{Analysis of EEG of MCI and AD patients}

Studies have shown that AD has (at least) three major effects on EEG (see [4] for an in-depth review): slowing of the EEG, reduced complexity of the EEG signals, and perturbations in EEG synchrony. Those effects, however, are not always easily detectable: there tends to be a large variability among AD patients. As a result, none of those phenomena allow at present to reliably diagnose $\mathrm{AD}$ at an early stage. Many recent studies have investigated how to improve the sensitivity of EEG for detecting AD. In the following we briefly review some of these studies; we will treat the three major effects of AD on EEG separately.

\subsection{Slowing of EEG}

Many studies have shown that Alzheimer's disease (AD) causes EEG signals to slow down (see, e.g., $[5,10,18-24,28])$ : AD is associated with an increase of power in low frequencies (delta and theta band, $0.5-8 \mathrm{~Hz}$ ) and a decrease of power in higher frequencies (alpha and beta, $8-30 \mathrm{~Hz}$, and gamma, 30-100Hz). To quantify the changes in spectral power, one has applied Fourier transforms $[5,10,18-22,28]$ and sparsified time-frequency maps ("bump models") [23, 24].

\subsection{Reduced Complexity of EEG Signals}

The EEG of MCI and AD patients seems to be more regular than of age-matched control subjects [6-8]. The following complexity measures have been used to quantify this reduction in EEG complexity: approximate entropy [6], auto mutual information [6], sample entropy [6,8], multiscale entropy [6], Lempel-Ziv complexity [6], and fractal dimension [7].

\subsection{Perturbations in EEG Synchrony}

Numerous studies have reported decreased EEG synchrony in MCI and AD patients under resting conditions ("spontaneous EEG")(see, e.g., [19,33,4558]). More precisely, the statistical dependence between spontaneous EEG signals recorded from different channels seems to be generally lower in MCI and $\mathrm{AD}$ patients than in age-matched control subjects. A large variety of measures have been applied to quantify this loss in statistical dependence, e.g., Pearson correlation coefficient [51], coherence [51, 52, 54], Granger causality [33, 45, 51], information-theoretic $[58,51]$ and state space based synchrony measures $[19,46$, $51,55,56]$, phase synchrony indices $[19,51,53,54,57]$, and stochastic event synchrony $[47-51]$. In a recent study, the spatial distribution of EEG phase synchrony in $\mathrm{AD}$ patients has been investigated [60]. By means of closely-related graph-theoretic methods, several studies have shown that EEG signals of MCI and $\mathrm{AD}$ patients have weaker small-world network characteristics compared to age-matched control subjects $[62,61]$.

The observed loss in synchrony is often attributed to a functional disconnection of the neocortex; it is probably not simply due to a loss of cortical neurons. 
For example, it may result from anatomical disconnections among different cortical regions in combination with reduced cholinergic coupling between cortical neurons [58]. In particular, a common hypothesis is that basal forebrain neurons may be severely affected in $\mathrm{AD}$, resulting in a cerebral cholinergic deficit that leads to memory loss and other cognitive symptoms [58].

Interestingly, in a few studies that investigate the EEG of MCI and $\mathrm{AD}$ patients recorded during working memory tasks, an increase of EEG synchrony was observed in those patients $[55,59]$. This inverse effect is often interpreted as the result of a compensatory mechanism in the brain.

One should keep in mind, however, that it is hard to directly interpret results obtained with synchrony measures. The synchrony of EEG signals may be significantly affected by brain events other than changes of synchrony, and by choices (like the reference electrode) that necessary have to be made during the analysis. Furthermore, as a single active source in the brain may affect the EEG signals across the entire scalp, changes in synchrony, and especially simultaneity of some events across channels, may be observed when the activity of one source alone changes, which is remote from a change in synchrony. As an alternative, one may use inversion methods to reconstruct sources, and next apply synchrony measures to those sources. However, the inversion problem is known to be notoriously difficult, and any source reconstruction method relies on certain assumptions, which may not necessarily hold.

\section{Discussion}

As pointed out earlier, EEG seems an attractive brain imaging modality for diagnosing $\mathrm{AD}$, since EEG recording systems are inexpensive and (potentially) mobile. Moreover, in contrast to most other non-invasive brain imaging methods, EEG has high temporal resolution, and may therefore contain crucial information about abnormal brain dynamics in $\mathrm{AD}$ patients.

Numerous studies have investigated the potential of EEG as a diagnostic tool for AD. At present, however, it is hard to assess whether EEG is truly useful for diagnosing AD. First of all, most studies report the results of statistical tests (p-values) without statistical post-correction. Since typically one conducts multiple tests simultaneously (sometimes hundreds or even thousands), e.g., individual pairs of electrodes or frequency bands, it is important to eliminate false positives. To this end, one may apply Bonferroni post-correction, or more powerful alternatives, such the false-discovery-rate correction method of Storey [65]. Unfortunately, not all studies on diagnosing AD from EEG signals apply such post-correction methods, and therefore, it is not always obvious how to interpret the reported results.

Second, few studies conduct discriminant analysis (linear or non-linear discriminant analysis, using support vector machines, neural networks, etc.); studies that do conduct discriminant analysis typically only report results for training data. The reported results are therefore often prone to overfitting, and they may be overoptimistic. To obtain more reliable classification results, one may for example apply crossvalidation, as has indeed been done in a handful studies (e.g., $[10,8,51])$. 
Third, in most existing studies, a single measure to detect EEG abnormalities is applied to a single EEG data set. Since almost every study considers a different measure and a different EEG data set, it is hard to compare existing studies and to verify whether results are consistent.

Fourth, it is likely that one will need to combine various EEG characteristics in order to obtain a good diagnostic tool for $\mathrm{AD}$, e.g., based on slowing, loss in EEG synchrony and complexity, and other features yet to be discovered. However, few studies systematically investigate large collections of EEG features (e.g., $[10,51])$; it would be of great interest to apply dimensionality reduction methods to hundreds or even thousands of EEG features, to determine the most discriminative EEG features in a disciplined and statistically rigorous fashion. Moreover, it still needs to be verified whether the effects listed in Sections 2.1 to 2.3 are independent. For example, it may be that EEG slowing and loss of EEG complexity are two sides of the same coin.

\section{Conclusions}

To conclude this paper, we point out several remaining challenges and topics for future research. At present, it is fairly difficult to gain access to EEG data of MCI or AD patients. Such databases are not publicly available, in contrast to ECG and other biomedical data (e.g., [66]). As a result, it is hard to systematically benchmark and assess the existing methods for diagnosing AD from EEG signals. Moreover, virtually none of those methods incorporate biophysical knowledge about AD (but see [37]); detailed mathematical models of the pathology of $\mathrm{AD}$, in conjunction with EEG data analysis, may help us to improve the diagnosis of AD. Along the same lines, one may expect further improvement by combining EEG with other imaging modalities, such as MRI (see, e.g., [31-33]), dMRI [34], TMS [35], and SPECT [36].

The correlation between $\mathrm{AD}$ risk factors (e.g., high plasma concentration of homocysteine [44]) and EEG characteristics needs to be investigated in greater detail. In addition, at present, the precise relation between the decline of cognition and memory and EEG abnormalities in AD patients remains largely unexplored (but see $[64,18,17,57]$ ). It is also of great importance to investigate whether EEG helps to distinguish between MCI and different stages of $\mathrm{AD}$ (see, e.g., [16]), and between $\mathrm{AD}$ and other dementias (see, e.g., [25-30]).

An important degree of freedom is the EEG recording condition: one may record EEG: (i) while the subject is at rest (with open or closed eyes); (ii) while the subject performs working-memory or other tasks; (iii) while the subject is being stimulated with auditory, visual, tactile, or other signals (see, e.g., [1115]). Depending on the recording situation, EEG signals may be more or less discriminative for MCI and $\mathrm{AD}$; a systematic exploration of different recordings conditions with the aim of diagnosing MCI and $\mathrm{AD}$ needs to be conducted.

One may also analyze the EEG of animal models of AD (see, e.g., [63]), where the progression of $\mathrm{AD}$ can be rigorously assessed and controlled; such studies may enable us to relate EEG abnormalities to the neuropathology of AD. Another interesting line of research is to investigate the effect of medication and therapy on the EEG of AD patients (see, e.g., [38-43]). 


\section{References}

1. Mattson M., 2004. Pathways towards and away from AD. Nature vol. 430.

2. Shimokawa et al., 2001. Influence of deteriorating ability of emotional comprehension on interpersonal behavior in Alzheimer-type dementia. Brain and Cognition 47(3): 423-433.

3. Weiner W.M., 2009. Editorial: Imaging and Biomarkers Will be Used for Detection and Monitoring Progression of Early Alzheimer's Disease. J. Nutr. Health Aging 4:332.

4. Jeong J., 2004. EEG Dynamics in patients with Alzheimer's disease. Clinical Neurophysiology 115, 1490-1505.

5. Baker et al., 2008. EEG Patterns in Mild Cognitive Impairment (MCI) Patients. Open Neuroimag J. 2: 52-55.

6. Hornero et al., 2009. Nonlinear analysis of electroencephalogram and magnetoencephalogram recordings in patients with Alzheimer's disease. Phil. Trans. R. Soc. A $367: 317-336$.

7. Besthorn et al., 1995. Parameters of EEG dimensional complexity in Alzheimer's disease. Electroencephalography and Clinical Neurophysiology 95:8489.

8. Woon et al., 2007. Techniques for early detection of Alzheimer's disease using spontaneous EEG recordings. Physiol. Meas. 28 335-347.

9. Park et al., 2007. Multiscale Entropy Analysis of EEG from Patients under Different Pathological Conditions. Fractals 15(4):399-404.

10. Besthorn et al., 1997. Discrimination of Alzheimer's disease and normal aging by EEG data. Electroencephalography and Clinical Neurophysiology 103:241-248.

11. Papaliagkas et al., 2008. Usefulness of event-related potentials in the assessment of mild cognitive impairment. BMC Neuroscience 9:107.

12. van Deursen et al., 2009. 40-Hz steady state response in Alzheimer's disease and mild cognitive impairment. Neurobiol Aging.

13. Caravaglios et al., 2008. Decreased amplitude of auditory event-related delta responses in Alzheimer's disease. Int. J. Psychophysiol. 2008 Oct;70(1):23-32.

14. van Deursen et al., 2009. Response speed, contingent negative variation and P300 in Alzheimer's disease and MCI. Brain Cogn. 69(3):592-9.

15. Güntekin et al., 2009. Decrease of evoked delta, theta and alpha coherences in Alzheimer patients during a visual oddball paradigm. Brain Res. 1235:109-16.

16. Giannakopoulos et al., 2009. Electrophysiological markers of rapid cognitive decline in mild cognitive impairment. Front Neurol Neurosci. 24:39-46.

17. Moretti et al., 2009. Increase of theta/gamma ratio is associated with memory impairment. Clin. Neurophysiol. 120(2):295-303.

18. van der Hiele et al., 2007. EEG correlates in the spectrum of cognitive decline. Clin Neurophysiol. 118(9):1931-9.

19. Czigler et al., 2008. Quantitative EEG in early Alzheimer's disease patients - power spectrum and complexity features. Int. J. Psychophysiol. 68(1):75-80.

20. Gianotti et al., 2007. Correlation between disease severity and brain electric LORETA tomography in Alzheimer's disease. Clin. Neurophysiol. 118(1):186-96.

21. Herrmann et al., 2005. Human EEG gamma oscillations in neuropsychiatric disorders. Clin. Neurophysiol. 116(12):2719-33.

22. Latchoumane et al., Multiway analysis of Alzheimers disease: classification based on space-frequency characteristics of EEG time series, 2008. Proc. World Congress on Engineering 2008.

23. Vialatte et al., 2008. Why sparse bump models? OHBM meeting, Melbourne, Australia, June 15-19 2008. Neuroimage, 41(S1):S159. 
24. Vialatte et al., 2005. Early Diagnosis of Alzheimer's Disease by Blind Source Separation, Time Frequency Representation, and Bump Modeling of EEG Signals. Proc. 15th International Conference on Artificial Neural Networks, Springer, LNCS 3696, pp. 683-692.

25. Jaeseung Jeong et al., 2001. Nonlinear dynamical analysis of the EEG in patients with Alzheimer's disease and vascular dementia. Journal of Clinical Neurophysiology 18(1): $58-67$.

26. Andersson et al., 2008. Electroencephalogram variability in dementia with lewy bodies, Alzheimer's disease and controls. Dement. Geriatr. Cogn. Disord. 26(3):28490.

27. Gawel et al., 2009. The value of quantitative EEG in differential diagnosis of Alzheimer's disease and subcortical vascular dementia. J Neurol Sci.

28. Schreiter et al., 2008. Alzheimer disease versus mixed dementias: an EEG perspective. Clin Neurophysiol 119(10):2255-9.

29. Bonanni et al., EEG comparisons in early Alzheimer's disease, dementia with Lewy bodies and Parkinson's disease with dementia patients with a 2-year follow-up. Brain. 131:690-705.

30. Uhlhaas et al., 2008. Visual perceptual organization deficits in Alzheimer's dementia. Dement Geriatr Cogn Disord. 25(5):465-75.

31. Babiloni et al., 2009. Hippocampal volume and cortical sources of EEG alpha rhythms in mild cognitive impairment and AD. Neuroimage 44(1):123-35.

32. Moretti et al., 2009. Increase of Theta/Gamma and Alpha3/Alpha2 Ratio is Associated with Amygdalo-Hippocampal Complex Atrophy. J Alzheimers Dis.

33. Babiloni et al., 2009. White-matter lesions along the cholinergic tracts are related to cortical sources of EEG rhythms in amnesic mild cognitive impairment. Hum. Brain Mapp. 30(5):1431-43.

34. Teipel et al., 2008. Regional networks underlying interhemispheric connectivity: An EEG and DTI study in healthy ageing and amnestic mild cognitive impairment. Hum Brain Mapp 30(7):2098-2119.

35. Julkunen et al., 2008. Navigated TMS combined with EEG in MCI and AD: a pilot study. J. Neuroscience Methods 172(2):270-6.

36. Musha et al., 2002. A new EEG method for estimating cortical neuronal impairment that is sensitive to early stage Alzheimer's disease. Clin. Neurophysiol. 113(7):1052-8.

37. Sotero R.C. and Trujillo-Barreto N.J., 2008. Biophysical model for integrating neuronal activity, EEG, fMRI and metabolism. Neuroimage 39(1):290-309.

38. Babiloni et al., 2009. Ibuprofen treatment modifies cortical sources of EEG rhythms in mild Alzheimer's disease. Clin. Neurophysiol. 120(4):709-18.

39. Gianotti et al., 2008. Rivastigmine effects on EEG spectra and three-dimensional LORETA functional imaging in Alzheimer's disease. Psychopharmacology (Berl) 198(3):323-32.

40. Yener et al., 2007. Increased frontal phase-locking of event-related theta oscillations in Alzheimer patients treated with cholinesterase inhibitors. Int. J. Psychophysiol. 64(1):46-52.

41. Kogan et al., 2007. Long-term tetrahydroaminoacridine treatment and quantitative EEG in Alzheimer's disease. J. Neural Transm. Suppl. (72):203-6.

42. Babiloni et al., 2006. Donepezil effects on sources of cortical rhythms in mild Alzheimer's disease: Responders vs. Non-Responders. Neuroimage 31(4):1650-65.

43. Moraes et al., 2006. The effect of donepezil on sleep and REM sleep EEG in patients with Alzheimer disease: a double-blind placebo-controlled study. Sleep 29(2):199-205. 
44. Babiloni et al., 2007. Homocysteine and electroencephalographic rhythms in Alzheimer disease: a multicentric study. Neuroscience 145(3):942-54.

45. Babiloni et al., 2009. Directionality of EEG synchronization in Alzheimer's disease subjects. Neurobiol Aging 30(1):93-102.

46. Kramer et al., 2007. Synchronization measures of the scalp EEG can discriminate healthy from Alzheimer's subjects. International Journal of Neural Systems 17, 1-9.

47. Dauwels J., Vialatte F., Rutkowski T., and Cichocki A., 2007. Measuring neural synchrony by message passing, Advances in Neural Information Processing Systems 20 (NIPS 20), in press.

48. Dauwels et al., 2009. Quantifying statistical interdependence by message passing on graphs, Part I: One-dimensional point processes. Neural Computation 21:8, in press.

49. Dauwels et al., 2009. Quantifying statistical interdependence by message passing on graphs, Part II: Multi-dimensional point processes. Neural Computation 21:8, in press.

50. Dauwels et al., 2008. Quantifying the Similarity of Multiple Multi-Dimensional Point Processes by Integer Programming with Application to Early diagnosis of Alzheimers Disease from EEG. Proc. 30th IEEE EMBC.

51. Dauwels et al., 2009. A comparative study of synchrony measures for the early diagnosis of Alzheimer's disease based on EEG. NeuroImage, in press.

52. Stevens et al., 2001. Dynamic regulation of EEG power and coherence is lost early and globally in probable DAT. Eur. Arch. Psychiatry Clin. Neurosci. 251, 199-204.

53. Koenig et al., 2005. Decreased EEG synchronization in Alzheimer's disease and mild cognitive impairment. Neurobiol. Aging. 26, 165-71.

54. Stam et al., 2007. Phase lag index: assessment of functional connectivity from multi channel EEG and MEG with diminished bias from common sources. Hum. Brain Mapp. 28(11):1178-1193.

55. Pijnenburg et al., 2004. EEG Synchronization likelihood in MCI and AD during a working memory task. Clin. Neurophysiol. 115, 1332-9.

56. Wan et al., 2008. Linear and nonlinear quantitative EEG analysis. IEEE Eng. Med. Biol. Mag. 27(5):58-63.

57. Park et al., 2008. Decreased EEG synchronization and its correlation with symptom severity in Alzheimer's disease. Neurosci Res. 62(2):112-7.

58. Jeong J., Gore J., and Peterson B., 2001. Mutual information analysis of the EEG in patients with Alzheimer's disease. Clinical Neurophysiology 112, 827-835.

59. Jiang Z. Y., 2005. Abnormal Corticol Functional Connections in AD: Analysis of Inter- and Intra-Hemispheric EEG Coherence. J Zhejiang Univ Sci B. 6, 259-264.

60. Knyazeva et al., 2008. Topography of EEG multivariate phase synchronization in early Alzheimer's disease. Neurobiol Aging.

61. He Y. et al., 2009. Neuronal Networks in Alzheimer's Disease. Neuroscientist.

62. Stam et al., 2007. Small-world networks and functional connectivity in Alzheimer's disease. Cereb Cortex 17(1):92-9.

63. Bobkova et al., 2008. Interhemispheric EEG differences in olfactory bulbectomized rats with different cognitive abilities and brain beta-amyloid levels. Brain Res. 2008.

64. Liddell et al., 2007. Rates of decline distinguish Alzheimer's disease and mild cognitive impairment relative to normal aging: integrating cognition and brain function. J. Integr. Neurosci. 6(1):141-74.

65. Storey J., 2002. A direct approach to false discovery rates. Journal of the Royal Statistical Society, Series B (Methodological) 64 (3): 479-498.

66. Goldberger et al., 2000. PhysioBank, PhysioToolkit, and PhysioNet: Components of a New Research Resource for Complex Physiologic Signals. 